\title{
Estudios de caso sobre buenas prácticas para superar el conflicto en el Valle del $\mathrm{Cauca}^{1}$
}

\author{
Luis Fernando Barón Porras \\ (Coordinador del proyecto)
}

\begin{abstract}
This paper summarizes the main findings of a research project that deals with five cases of best practices to overcome the conflict in Valle del Cauca. The terms of references for this project have been provided by the Bank of Best Practices to Overcome the Conflict established and maintained by the United Nations Development Program. The cases examined included the returning of the displaced persons to the places of their permanent residence, the fomenting of peace culture and the strengthening of local community, the regional TV channel efforts to disseminate relevant information, peace education for former rebels, and the promoting of legal agricultural activities in the coffee-producing area. Besides providing an overview of the main features of all of the cases, this report presents a summary of the socio-political and historical context in which these five experiences occur and reflects the lessons and recommendations that can be derived from a comparative analysis of the cases.
\end{abstract}

\section{Presentación}

El presente reporte de investigación presenta una síntesis de los resultados y análisis realizados como parte del desarrollo de cinco estudios de caso de experiencias del Valle del Cauca, desde la perspectiva establecida por el Banco de Buenas Prácticas para Superar el Conflicto del Programa de las Naciones Unidas para el Desarrollo (PNUD). El trabajo fue adelantado por investigadores del CIES, Centro de Investigación de la Facultad de Derecho y Ciencias Sociales de la Universidad Icesi. La selección de los casos en cuestión estuvo precedida por un proceso de identificación colectiva adelantado por el PNUD en el que,

1 Este informe es resultado de un trabajo colectivo desarrollado por el CIES, Centro de investigación de la Facultad de Derecho y Ciencias Sociales de ICESI con el apoyo del Banco de Buenas Prácticas para superar el conflicto de PNUD. En este trabajo participaron como investigadores principales Rosa Bermúdez, Enrique Jaramillo, Ana Lucía Paz y Vladimir Rouvinski y, como asistentes de investigación, Lady Otalara y Maritza Valencia. 
junto con actores locales, se seleccionaron 300 iniciativas de cinco regiones geográficas del país: los departamentos de Cauca, Valle, Antioquia, Santander y en la región de los Montes de María. Dentro de los criterios de identificación se valoraron los siguientes aspectos: pertinencia, eficacia, sustentabilidad, legitimidad y replicabilidad.

Para la región del departamento del Valle del Cauca, el grupo de investigadores de Icesi tuvo a su cargo el estudio de cinco experiencias: el proceso de retorno y fortalecimiento de la comunidad del Bajo Calima (realizado por Enrique Jaramillo); los programas de cultura para la paz y de fortalecimiento del gobierno local en las comunidades del río Cajambre (elaborado por Rosa Bermúdez); el programa de televisión Doce del Día - D3, del canal regional Telepacífico (hecho por Ana Lucía Paz); el programa de Estudios Políticos y Resolución de Conflictos de la Universidad del Valle (realizado por Vladimir Rouvinski), y el programa de Jóvenes Agricultores del Valle del Cauca desarrollado por el Comité Departamental de Cafeteros en asocio con la Cooperativa de Centrales Agrarias del Valle, CENCOA (estudio llevado a cabo por Luis Fernando Barón).

Las investigaciones, en conjunto, apuntan a reconstruir un contexto regional que ponga en relación las metodologías y acciones de cada práctica con las motivaciones y factores del entorno que las posibilitaron y determinaron. De esta manera, los estudios propenden por explicar la cadena de eventos, los contextos y las formas en las que se desarrollaron los procesos y que hacen viable o no para cada caso hablar de una buena práctica para la superación del conflicto. En palabras del documento de referencia del proyecto, se trata de responder a las preguntas: ¿cuáles son los ingredientes básicos o mínimos que identifican una acción colectiva como buena práctica? $\mathrm{O}$, si se quiere: ¿cuáles son las condiciones que hacen viable una buena práctica para superar el conflicto?

Este informe está estructurado en tres partes. En la primera se presenta una síntesis de los contextos de las cinco experiencias del departamento, acompañada de hipótesis preliminares sobre hechos transversales en la región. La segunda parte hace una presentación sintética de cada uno de los casos. Y finalmente, en la tercera parte se abordan las claves, lecciones y recomendaciones surgidas de un análisis comparativo de las cinco experiencias.

\section{Contexto}

Los contextos desarrollados en cada uno de los estudios de caso hacen posible identificar una serie de hechos, fenómenos y procesos que se entrecruzan y que permiten esbozar unas primeras hipótesis sobre momentos claves en la región, que por supuesto, es necesario estudiar y analizar con mayor profundidad. 
Un primer momento está relacionado con la preeminencia y continuidad de los conflictos agrarios, producto de procesos de colonización y de la lucha por la tierra y por los territorios, como ejes del conflicto armado en el departamento. Un segundo momento está representado por los procesos organizativos y de movilización social que antecedieron y precedieron la Constitución de 1991 y que muestran un énfasis en la búsqueda y en la presión por la inclusión política, cultural y étnica. El tercer momento lo constituye el escalamiento de la violencia, el conflicto armado y el desplazamiento en el Valle del Cauca en el período comprendido entre 1998 y 2004, producido por la expansión del narcotráfico, la lucha por corredores naturales y la llegada de los paramilitares. Finalmente, el cuarto momento está delineado por las respuestas de las organizaciones sociales, las instituciones locales, los medios de comunicación, la academia y sectores del empresariado a la grave crisis del período enunciado antes. Las cinco experiencias de este informe hacen parte de esas respuestas.

\section{Descripción de los casos}

\section{Proceso de retorno y fortalecimiento de la población de la cuenca baja del río Calima: atención a las victimas del conflicto}

Durante los primeros meses del año 2003 gran parte de la población afrodescendiente que habita la cuenca baja del río Calima, sector rural del municipio de Buenaventura, se vio obligada a desplazarse hacia distintos lugares de la región, debido a los hostigamientos y las acciones de guerra de grupos armados al margen de la ley. Desde el mismo momento de su desplazamiento, la población, hacinada en casas de alquiler y casas de familiares o amigos, emprende a través del Consejo Comunitario acciones tendientes a la construcción de un proceso de retorno que culminará en septiembre de 2004 con el regreso efectivo de la mayoría de los habitantes y el comienzo de diferentes estrategias de fortalecimiento de la comunidad. Este proceso, que se dio de forma gradual, contó en diferentes momentos con el apoyo de las autoridades civiles del municipio y del departamento (especialmente la Oficina de Gestión de Paz), al igual que el acompañamiento de entidades y organismos nacionales e internacionales.

El retorno representa para la comunidad una forma de reconstrucción de los lazos Sociedad-Estado. En efecto, el capital social adquirido en este reencuentro traumático con la sociedad le ha garantizado a la comunidad cierta autonomía respecto a los actores armados, negociando pactos y aprendiendo nuevas reglas de juego en un ambiente siempre cambiante. Hoy en día no se puede decir que el retorno ha sido exitoso porque la gente esté restablecida en condiciones sociales y humanitarias plenas, ni porque las intervenciones institucionales hayan logrado 
contrarrestar por completo los efectos del conflicto social y armado. Lo que sí se puede decir es que ha sido exitoso porque la gente no se ha devuelto. En este sentido, un trabajo de campo más extenso con una co-residencia prolongada debería permitir analizar, más allá del nivel de las organizaciones, las formas de acción que los sujetos en su día a día logran desarrollar para hacer frente a los contextos cambiantes del conflicto.

\section{Cultura para la paz en el río Cajambre: fortalecimiento del Estado local}

La experiencia de la población del río Cajambre, ubicada en el sector sur del Puerto de Buenaventura (Valle del Cauca), muestra las acciones colectivas adelantadas por la sociedad civil para enfrentar el conflicto armado, con la particularidad de tratarse de una comunidad étnica de negros afrodescendientes que llegaron al territorio en 1786 como esclavos de las minas de oro. En la actualidad esta población, de aproximadamente 4.000 pobladores en la cuenca, hace parte de un movimiento social a nivel regional que exige derechos étnico-territoriales y su determinación de neutralidad y autonomía frente a los actores armados. Este caso se analiza desde cuatro ámbitos centrales que marcan el desarrollo del proceso: la re-invención de lo étnico como reivindicación política, la gobernabilidad y autoridad local (imaginarios y representaciones), la sociedad civil frente a los actores armados (la exigencia a la libre determinación), la gestión social del territorio (educar para la paz).

En la región se vive un proceso de disputa intensa del territorio en el que la gobernabilidad y el control territorial se encuentran interpelados. Han sido múltiples las situaciones violentas entre la guerrilla, los grupos paramilitares y el ejército de Colombia que han producido múltiples desplazamientos. Para la población del río Cajambre las acciones armadas que presionan su desplazamiento son vistas como una estrategia para usurpar sus territorios, asumiendo que se trata de un fenómeno que persigue el destierro, la desaparición de las poblaciones y la generación de una dinámica cotidiana del terror y del miedo, desestructurando el tejido social y los procesos organizativos étnico territoriales.

Por eso el pueblo cajambreño ha adelantado acciones concretas mediante alternativas productivas a partir del manejo sostenible del territorio, la biodiversidad y los recursos naturales que se han hecho tangibles en la formulación del Plan de Manejo Territorial (PMT) desde el año de 1999, en el cual se abordaron las siguientes áreas estratégicas: fortalecimiento organizativo, ordenamiento territorial y seguridad alimentaria, gestión e investigación. El Consejo Comunitario implementa este plan cotidianamente a través de la educación y la construcción de consensos y acuerdos sociales, garantizando un control real sobre el territo- 
rio colectivo. Este caso muestra con detalle el desarrollo de estas acciones en defensa de la autonomía, el autogobierno y la ciudadanía.

\section{Jóvenes agricultores del Valle del Cauca: prevención del reclutamiento}

El programa Jóvenes Agricultores (JAV) nace en medio de la crisis del café del año 1997, la cual se recrudece por la desaceleración económica nacional de 1999, y surge como respuesta a dos problemas identificados por el gremio cafetero: el bajo relevo generacional en el sector y la migración de los jóvenes campesinos hacia la ciudad. El programa desarrollado por el Comité Departamental de Cafeteros del Valle en asocio con la Cooperativa de Centrales Agrarias del Valle del Cauca, CENCOA, se propone como un modelo experimental de reforma agraria en zonas rurales del Valle del Cauca que involucra familias jóvenes campesinas para otorgarles tierras productivas para el cultivo del café y algunas para la caña de azúcar. Las fincas son distribuidas entre los beneficiarios con un promedio de cinco hectáreas por familia. Sin embargo, el programa no se limita a entregar la tierra, sino que brinda asesoría técnica, social y administrativa por medio del apoyo y acompañamiento permanente. El acompañamiento se mantiene por un tiempo prudencial mientras los nuevos caficultores pueden hacerse cargo de sus fincas.

Esta experiencia ha desarrollado y probado un modelo que aporta herramientas para la reflexión y la búsqueda de alternativas para el desarrollo de una reforma agraria, desde una visión renovada de la misma. Igualmente las trayectorias y aprendizajes del programa y de los jóvenes que lo componen han mostrado que ésta es una iniciativa efectiva para prevenir y desestimular el reclutamiento y la vinculación de jóvenes a los grupos armados ilegales y a actividades ilícitas y criminales. Las claves de esta experiencia están en una serie de asuntos interrelacionados, a saber: en el fortalecimiento de la autoestima y la identidad campesina y cafetera de los jóvenes; en el restablecimiento de la confianza vecinal y en el desarrollo y reorganización de espacios de trabajo y acción comunitaria; en el desarrollo de proyectos agrícolas que, además de contribuir a la seguridad alimentaria, son viables y sustentables; en la reactivación de circuitos económicos comunitarios y locales, y en el reconocimiento social y en el soporte brindado por la Federación Nacional de Cafeteros y su vínculo con las organizaciones cafeteras del departamento como CENCOA. 


\section{Programa D3 de Telepacifico: comunicación para la pazy la convivencia}

El programa de televisión D3 de Telepacífico se denomina de esa manera desde el año 2004, pero tiene sus antecedentes en programas anteriores que surgen junto con la creación del canal a finales de la década de los ochenta. Lo anteceden los programas "Punto de Encuentro", "Desaparecidos" y "Desaparecidos y Desplazados". Desde su origen fue cofinanciado entre Telepacífico y la Comisión Nacional de Televisión (CNTV) y sale del aire en enero de 2008 por cambios en la política de programación del canal.

D3 contribuyó a visibilizar el tema del conflicto desde dos perspectivas básicas y diferenciadas. Por una parte se diseñaron y emitieron programas sobre el conflicto que fueron trabajados por el director e invitados expertos en el tema. Y por otra parte, se abrió un espacio a los ciudadanos para presentar sus casos, hacerlos visibles y obtener información sobre mecanismos de búsqueda de desaparecidos. Si bien D3 no tuvo como tema central los desaparecidos, heredó de los programas anteriores el foco sobre el tema y, al menos, se presentaron algunos casos al público. Esto convirtió al canal en una instancia potencial de construcción de lo público, un lugar simbólico que recogía los intereses y sensibilidades de los ciudadanos y ponía el tema en la agenda pública, acercándola a estos temas sensibles.

El programa permitió que la comunidad dispusiera de información confiable y oportuna sobre las instancias e instituciones que resuelven problemas. Aunque no hay evidencia empírica contundente que refleje la construcción de una red interinstitucional de atención a víctimas del conflicto, sí se reconoce un interés permanente del programa por emitir información que ayude a conocer procedimientos relativos a la atención de víctimas, como denuncias y atención a los desplazados, etc. El programa fue un nodo de intercomunicación entre la comunidad y las instituciones y de la comunidad entre sí. Esto une a los ciudadanos alrededor de sus vivencias y de las acciones para enfrentarlas.

\section{Programa de Estudios Políticos y Resolución de Conflictos: educación para la pazy la convivencia}

Después de la constitución de 1991 y la desmovilización y entrega de armas de diferentes grupos guerrilleros como el M-19, el Quintín Lame, el EPL, algunos bloques del ELN, entre otros, surge la necesidad de programas que vinculen a estos individuos denominados como "victimarios" en procesos de educación para la paz, con el fin de lograr no sólo la reinserción de estos desmovilizados, sino además una integración política, ya que algunos de los ex-combatientes empezaban a ocupar puestos de reconocimiento social y político tanto en la esfera pública como en la privada. 
En este contexto, y como producto de un trabajo conjunto entre la Compañía Nacional para la Paz (COMPAZ, organización creada por excombatientes del M-19) y la Universidad del Valle, nace el programa de pregrado de "Estudios Políticos y Resolución de Conflictos", el cual comenzó en el año 1997 dentro del Instituto de Altos Estudios Jurídicos y Políticos de la universidad. Dicho programa estaba dirigido no sólo a guerrilleros desmovilizados del M-19 sino a todos los ex-militantes que hicieron parte de procesos de paz con los gobiernos colombianos. El programa es la única experiencia de profesionalización en el área de ciencias sociales en Colombia dirigida a ex-combatientes

Esta experiencia permitió que los ex-militantes de diferentes grupos guerrilleros, en especial los del M-19, continuaran en la búsqueda y en el ejercicio de la política, pero de manera legal y legítima para la sociedad. Los egresados, con mejor formación y siendo más competitivos, podían optar por puestos políticos durante y después del proceso, como gestores de paz, funcionarios de los gobiernos locales y regionales, diputados y concejales. Pero el programa no sólo benefició a los ex-combatientes en términos profesionales, también lo hizo en el plano personal. Esta experiencia significó un reconocimiento simbólico de sus experiencias, que no quedaron en el olvido. Más cuando se sabe que gran parte de sus experiencias como combatientes son rechazadas por la sociedad e incluso por sus entornos más cercanos como sus familias o sus vecinos. Este reconocimiento simbólico es uno de los factores que facilitó significativamente el proceso de reinserción.

\section{Claves regionales para superar el conflicto en Colombia}

Señalar claves en términos de buenas prácticas para superar el conflicto a partir del análisis de las experiencias estudiadas es un ejercicio que implica precisar que no es posible generalizarlas, en el sentido de constituir aspectos universales válidos en cualquier circunstancia. Por el contrario, un primer aspecto que interesa resaltar es que la importancia que adquieren los diversos factores que intervienen en las prácticas está dada por el conjunto de las mismas, por su sinergia en cada caso particular, en la que se han ido configurando como un constructo social - es decir, los contextos socio-históricos en los que aparecen. En las experiencias analizadas es más apropiado hablar de claves para enfrentar el conflicto. En estas experiencias encontramos diversas estrategias agenciadas desde fortalezas culturales, institucionales, sociales y políticas en los diversos contextos en los que el conflicto armado en la región se desarrolla. Estas experiencias nos permiten señalar diversas dinámicas y lógicas bajo las cuales agentes estatales, comunitarios, gremiales y sociales están construyendo alternativas para atenuar las consecuencias de la guerra. 
Así, la prevención del reclutamiento de jóvenes en las zonas de economía cafetera, la atención a la población desplazada por las confrontaciones armadas entre los actores, el apoyo a los familiares de las personas desaparecidas para establecer puntos de encuentro y sensibilizar la audiencia con respecto al sufrimiento social ocasionado por las consecuencias de la guerra, la objeción cultural de las minorías étnicas para no participar del conflicto armado exigiendo su derecho de neutralidad en el conflicto y el respeto a su territorio colectivo, constituyen algunos de estos esfuerzos que ofrecen sugerencias de aprendizajes sociales para encontrar caminos alternos en la construcción de una sociedad más justa y humana. De igual forma, el análisis de estas experiencias sugiere aspectos relevantes que, de ser desarrollados, mostrarían con plenitud su potencialidad, lo que ya es un elemento de aporte significativo para indicar caminos necesarios de recorrer en la superación del conflicto en el país. En este sentido, también es importante entender las claves identificadas en el ejercicio realizado. No sólo es necesario señalar lo que no se ha hecho, sino que resulta importante mostrar la dimensión y el impacto de las carencias presentadas.

Un primer asunto a destacar en los estudios de caso realizados está en función de entender las lógicas colectivas de los grupos sociales en juego. Aquí es interesante resaltar la idea central que aparece en los casos acerca de la identidad (compartida, social, cultural y política), que hace posible hablar de acción colectiva, de movimiento social, de acción de grupo, entre otros. En el caso de las minorías étnicas, este es un aspecto central para el desarrollo de la acción colectiva de defensa y resistencia. En el caso de los jóvenes caficultores, se manifiesta en su arraigo en la subjetividad presente en el ser "campesino", en el trabajar la tierra, en ser caficultor. De igual forma, aparecen identidades construidas en las opciones de vida, como en el caso de los ex-combatientes o desmovilizados que funcionan como grupo con ideales políticos compartidos en su condición de reinsertados en una sociedad que les ofrece a través del programa de formación política una opción que sigue siendo válida socialmente para sujetos políticos.

Estrechamente articulado con lo anterior está el asunto del reconocimiento social y político, de la visibilización y la inclusión social de los diferentes actores en la confrontación política. En gran parte, el éxito de los casos estudiados consiste en haber creado espacios de reconocimiento del otro en su condición, en haber abierto las puertas de la inclusión política y social a poblaciones marginadas y/o estigmatizadas. En este sentido, podría afirmarse que la inclusión social, política, simbólica y cultural constituye una de las claves más importantes para superar el conflicto armado en el país. 
De otra parte, los casos analizados muestran aspectos interesantes en la forma como la relación Estado-Sociedad se entiende en cada uno de ellos, en el contexto del conflicto y en las alternativas para enfrentarlo. En este sentido, un aspecto que resulta importante resaltar son los desarrollos normativos y jurídicos que ofrecen garantías a la población para defender sus derechos: en este caso, de manera central, el derecho a la vida y los derechos colectivos, como los de las minorías étnicas. En la medida en que la población hace un uso de estas herramientas legales y las siente suyas, ha tenido mejores resultados en sus acciones para enfrentar las consecuencias del conflicto.

En los casos analizados, otro aspecto que resulta altamente significativo es el peso que tiene el fortalecimiento de la actuación institucional, como espacios que han alcanzado cierta legitimidad social y donde se construye aquello que llamamos "lo público". Así, las organizaciones sociales, las instancias gubernamentales y las dinámicas gremiales, son escenarios privilegiados para el debate público y la acción mediadora. Las estrategias para enfrentar el conflicto requieren que las instituciones cumplan una función central en la dinamización de lo público, en la promoción del ejercicio ciudadano y en la facilitación de la interlocución de los diferentes actores. Así, las definiciones normativas, con las dinámicas institucionales logran efectivamente constituirse en referentes de autoridad y establecimiento de los órdenes sociales.

En igual sentido, se puede leer la actuación de la cooperación internacional como marco institucional que fortalece la acción ciudadana. Es importante resaltar que en lso casos de las poblaciones más marginales y menos protegidas -Bajo Calima y Cajambre- el acompañamiento institucional internacional a través de las agencias de cooperación ha sido imprescindible para la visibilización del conflicto y de la situación de exclusión de la población, y para la configuración de alternativas de atención en medio del conflicto. El papel jugado por las organizaciones de cooperación internacional también indica las debilidades de la sociedad civil para enfrentar a los actores armados y exigir el cumplimiento de sus derechos, así como también evidencia las debilidades estatales para el ejercicio de gobernabilidad y protección de la población en medio de la confrontación armada en el país. No obstante, la actuación de estas organizaciones también se constituye en una forma de ganar legitimidad en estos procesos, tanto por parte del Estado que en la alianza se expone ante la mirada internacional, como de las organizaciones sociales (sociedad civil en general) que buscan mayor protección y garantías de cumplimiento y le apuestan a una presencia y participación en mejores condiciones de seguridad y con mayores probabilidades de eficacia.

De igual forma, la apertura formal en espacios institucionales de asuntos centrales de las dinámicas del conflicto -como la difusión de la situación de las personas desplazadas y desaparecidas en el programa D3 emitido a través del 
canal de televisión regional- coloca de manifiesto la proyección que adquiere este tratamiento a la problemática cuando se ejerce desde lugares estratégicos para la construcción de lo público como lo son los medios de comunicación: un ejercicio ciudadano para la visibilización del conflicto y sus consecuencias. Una aproximación en este mismo sentido puede sugerirse en el caso del programa de formación para la paz adelantado por la Universidad del Valle, colocándose en evidencia cómo la apertura y flexibilidad institucional ofrecen condiciones favorables e imprescindibles para el desarrollo de estas alternativas.

De otro lado, aunque con mayor difusión y análisis pero no de menor importancia, están aquellos factores de orden estructural y económico que han estado tan presentes en las explicaciones de las dinámicas del conflicto político. Los casos estudiados señalan el valor que aún tiene la confrontación social con respecto al modelo económico hegemónico como uno de los ejes centrales del conflicto social. Hay aquí una dimensión estructural económica del conflicto -la historia de la pobreza, desigualdad y carencia material- que sigue estando presente en sus dinámicas actuales, pero que ahora se expresa como oposición a la confrontación y busca opciones diferentes para su resolución.

En su conjunto, los casos analizados tienen una lección que podría asumirse como una lectura de la sociedad contemporánea que se rebela ante la historia de guerra y confrontación, para buscar caminos más “civilizados” y menos costosos en términos de vidas humanas y sufrimiento social. Estos nuevos caminos nos ponen en la perspectiva de la formación ciudadana para la resolución dialogada, negociada, pactada de nuestras diferencias, de nuestras divergencias, también de nuestras carencias. Se trata de que por distintos caminos de inclusión social, política, económica, simbólica y cultural se reconstruyan tejidos sociales en el horizonte. 